\title{
Thermophysical properties of nanofluids
}

\author{
Valery Ya. Rudyak ${ }^{1}$ and Andrey V. Minakov ${ }^{2}$ \\ 1 Novosibirsk State University of Architecture and Civil Engineering, Leningradskaya str. 113, Novosibirsk, 630008, Russia \\ 2 Siberian Federal University, Svobodniy av. 71, Krasnoyarsk, 660041, Russia
}

Received: date / Revised version: date

\begin{abstract}
This paper discusses the current state of knowledge of the thermophysical properties of nanofluids. The viscosity, thermal conductivity and heat transfer of nanofluids are considered. Experimental and molecular dynamics data are presented. It is shown that viscosity and thermal conductivity of nanofluids generally cannot be described by classical theories. The transport coefficients of nanofluids depend not only on the volume concentration of the particles but also on their size and material. The viscosity increases with decreasing the particle size while the thermal conductivity increases with increasing the particle size. The reasons for this behavior are discussed. The heat transfer coefficient is determined by the nanofluid flow mode (laminar or turbulent). The use of the nanofluids as a coolant significantly affect on the magnitude of the heat transfer coefficient. In laminar flow the heat transfer coefficient of nanofluids in all cases are much more than that of based fluid. It is shown that a $2 \%$-nanofluid intensifies the heat exchange more than twice compared to water. The effect of using nanofluids in turbulent mode depends not only on the thermal conductivity of nanofluid, but also on its viscosity.
\end{abstract}

PACS. PACS-key nanofluids - PACS-key discribing text of that key

\section{Introduction}

Nanofluids are two-phase systems consisting of a base fluid and nanoparticles. Typical carrier fluids are water, organic liquids (ethylene glycol, oil, biological liquids, etc.), and polymer solutions. The dispersed solid phase is usually nanoparticles of chemically stable metals and their oxides. The nanofluid studying was begun nearly twenty years ago.Continuously growing interest to nanofluids is due, to several reasons, first, due to their numerous existing and possible applications (in different chemical processes, biotechnologies, cooling of various devices, developing new systems for thermal energy production and transportation, new pharmaceutical, medicinal, and cosmetic products, systems for detection of impurities of various types and air and water purification, new lubricants, paints and varnishes, drug delivery systems, etc.). The transport processes are key processes because all applications of nanofluids are connected with their flows. Dozens of research groups around the world have studied these processes, and hundreds of papers have been published. However, the results of these studies were extremely controversial. It has been found that neither the viscosity nor the thermal conductivity of nanofluids can be described by classical theories (Einstein, Maxwell, etc.). Therefore, the study of nanofluids is of great importance for constructing a consistent theory of transport processes of condensed matter.

$\overline{\text { Send offprint requests to: }}$
The contradictory character of the experimental data obtained while measuring the thermophysical characteristics of nanofluids needed additional systematic measurements. Such experiments were carried out by the authors during the last two years. The description of the obtained results is the primary goal of this paper. The viscosity and thermal conductivity of more than fifty nanofluids have been studied. Nanofluids based on water, ethylene glycol, and engine oil with diamond, $\mathrm{SiO}_{2}, \mathrm{Al}_{2} \mathrm{O}_{3}, \mathrm{ZrO}_{2}, \mathrm{TiO}_{2}$, and $\mathrm{CuO}$ particles have been considered. The nanoparticles volume concentration ranged from 0.25 to $8 \%$. The particle size ranged from 5 to $151 \mathrm{~nm}$.

Measurements of the thermophysical properties of nanofluids provide only integral information about their transport processes and as a rule do not clarify the mechanisms of these processes. The mechanisms of transport processes were studied using the molecular dynamics method. Analysis of the mechanisms of the transport processes in nanofluids is the second goal of the paper. In the molecular dynamics simulation a standard molecular dynamics method was employed (see, e.g., [1]). A cubic cell and periodic boundary conditions were used. The interaction between the molecules of the carrier fluid was described by the Lennard-Jones potential. The interactions between basefluid molecules and a nanoparticle and nanoparticles each other were described using specially constructed potentials $[2,3]$.

Methods for obtaining nanofluids are not discussed in this paper. It was used so named two-step method. The 
required amount of nanopowder was first added to a base fluid, after which the disperse system was stirred mechanically and subjected to ultrasonic treatment to destroy nanoparticle agglomerates. In our experiments, the ultrasonic treatment continued until the measured viscosity or thermal conductivity ceased to change with increasing treatment time. In addition, each measurement was repeated several times, in particular, not on the same day.

\section{Viscosity of nanofluids}

The viscosity of suspensions was first studied by Einstein in his classical work [4]. Considering the motion of a single particle in a fluid, he determined the flow field perturbations caused by it, calculated the effective stress tensor, and obtained the following simple expression for the effective viscosity coefficient

$$
\eta=\eta_{0}(1+2.5 \varphi)
$$

Thus, the viscosity coefficient of a coarse suspension $\eta$ is always greater than the viscosity $\eta_{0}$ of the base fluid and depends only on volume concentration of dispersed particles $\varphi$. Comparison with experiment has shown that formula (1) satisfactorily describes the viscosity of suspensions with small volume concentrations of particles $\varphi \leq 10^{-2}$. At moderate particle concentrations (approximately to $10-15 \%$ ), the interaction between the particles should be taken into account. For this purpose, formula (1) has been modified in many studies (see, e.g., [5-11]). The modified formulas can be represented as

$$
\eta=\eta_{0}\left(1+2.5 \varphi+k \varphi^{2}\right),
$$

where the coefficient $k$ varies from 4.3 to 7.6.

The viscosity of nanofluids has been extensively investigated over twenty years in more than thirty groups throughout the world. However, a universal formula that would describe the viscosity coefficient of any nanofluid has not been derived. Moreover, measurements have often led to diametrically opposite results. Why does this occur?

It has long been thought that similarly to the viscosity of conventional suspensions, the viscosity of nanofluids is determined only by the volume concentration of particles. It is noteworthy that in special benchmark measurements made as part of an international project on viscosity and thermal conductivity measurements (International Nanofluid Properties Benchmark Exercise) nanofluid viscosity was also examined only as a function of particle volume concentration [12].

It has been reliably established that the nanofluid viscosity does not obey Einstein's formula (1) even at low particle concentrations. In all cases studied the nanofluid viscosoty is mach higher than the viscosity of coarse dispersed fluid. The viscosity can also be described by the linear relation $\eta=\eta_{0}(1+a \varphi)$, but, in this case, coefficient $a$ varies from 4.3 to 22 , depending on the type of nanofluid (see, e.g., $[11-17]$ ); i.e., it is several times the value predicted by the Einstein's theory.
In all cases, as the volume (or mass) concentration of nanoparticles increases, a quadratic dependence of the viscosity on $\varphi$ is obtained

$$
\eta=\eta_{0}\left[1+a \varphi+b \varphi^{2}\right]
$$

However, similarly to the coefficient $a$ (low concentration), the coefficient $b$ is not universal and far exceeds that for conventional suspensions. Several correlations obtained at different times are given below. One of the first correlations was obtained for a nanofluid with $\mathrm{TiO}_{2}$ particles [18]

$$
\eta=\eta_{0}\left[1+5.45 \varphi+108.2 \varphi^{2}\right] .
$$

A year later, the following experimental correlation was proposed for a water based nanofluid containing $\mathrm{Al}_{2} \mathrm{O}_{3}$ nanoparticles [19]

$$
\eta=\eta_{0}\left[1+7.3 \varphi+123 \varphi^{2}\right] .
$$

It is worth noting that in the same paper, a different correlation was proposed for a suspension of the same nanoparticles in ethylene glycol

$$
\eta=\eta_{0}\left[1-0.19 \varphi+306 \varphi^{2}\right] .
$$

There are more examples, but even these few data are sufficient to show that the obtained correlations are not universal. In contrast, the Einstein's formula for conventional suspensions is universal for all fluids and depends only on the volume concentration of particles. What are the reasons for the lack of universality for nanofluids? There may be two possible reasons. The viscosity of nanofluid may depend on the nanoparticle size and material.

Dependence of nanofluid viscosity on particle size was first predicted by molecular dynamic simulations [20] (see also [21]). It was found that the viscosity of nanofluid with small particles (from 1 to $2 \mathrm{~nm}$ ) decreases with increasing particle size. At nearly the same time, the dependence of nanofluid viscosity on particle size was confirmed experimentally [15]. However, studies of the relationship between viscosity and nanoparticle size are very few in number (only about one-quarter of the total number of publications dealing with nanofluid viscosity, according to review [13]). This is not surprising since until recently there have been very contradictory opinions regarding the dependence of viscosity on the particle size. For example, Prasher et al. [22] conclude that the viscosity of nanofluids is nearly independent of nanoparticle size. On the other hand, it is noted in $[17,23]$ that the nanofluid viscosity increases with increasing particle size. Namburu [15] was one of the first to show experimentally that the nanofluid viscosity increases with decreasing particle size. The same conclusion was reached in [24].

To resolve these contradictions, we initiated serial measurements of nanofluid viscosity as a function of particle size. The measurements were performed using different Brookfield viscometers at a controlled temperature, and their accuracy was $1-2 \%$. In the first series of experiments 


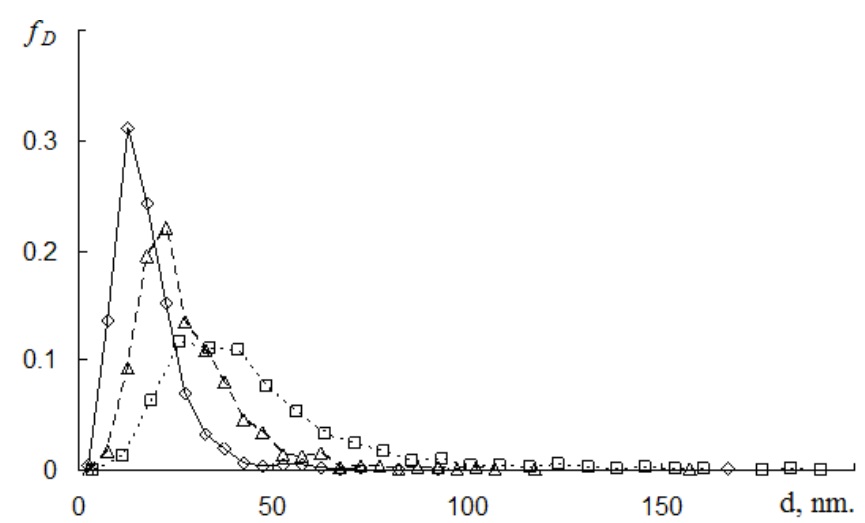

Fig. 1. Differential size distributions of $\mathrm{SiO}_{2}$ nanoparticles.

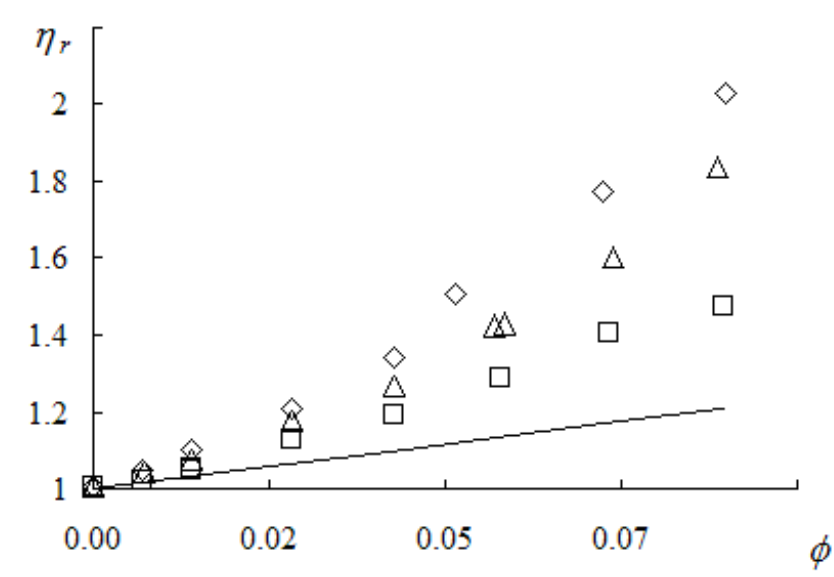

Fig. 2. Relative viscosity of ethylene glycol based nanofluid with $\mathrm{SiO}_{2}$ particles versus the volume concentration.

the dependence of the viscosity of nanofluids based on ethylene glycol with $\mathrm{SiO}_{2}$ particles with an average particle size of $18.1,28.3$, and $45.6 \mathrm{~nm}$ was studied. The volume concentration of the particles was varied from $0.25 \%$ to about $8 \%$. Differential particle size distributions in the nanofluids are shown in Fig. 1 [25]. Here rhombuses correspond to particles with an average size of $18.1 \mathrm{~nm}$, triangles to $28.3 \mathrm{~nm}$, and squares to $45.6 \mathrm{~nm}$. In all cases, these distributions were found to be lognormal. The obtained dependences of the relative viscosity $\eta_{r}=\eta / \eta_{0}$ on the volume concentration of nanoparticles are presented in Fig. 2. Here the symbols are the same as in Fig. 1 and the line corresponds to the viscosity coefficient according to Einsteins equation (1). The viscosity of all the three fluids considered are different and mach higher of the value predicted by formulas (1) or (2).

The data shown in Fig. 2 clearly indicate that the nanofluid viscosity increases with decreasing particle size. Nevertheless, to remove any doubt, we measured the viscosities of more than fifty nanofluids with different oxide particles. Some of these data are shown in Fig. 3 for the water based nanofluids. In all cases the volume concentrations of the particle are equal to $2 \%$ (see also the Table 1 ). The results of these measurements unambiguously suggest
Table 1. Relative viscosity coefficient of water based nanofluids depending on the particle concentration

\begin{tabular}{|l|l|l|l|l|l|}
\hline & $\begin{array}{l}\mathrm{Al}_{2} \mathrm{O}_{3} \\
(150 \mathrm{~nm})\end{array}$ & $\begin{array}{l}\mathrm{TiO}_{2} \\
(150 \mathrm{~nm})\end{array}$ & $\begin{array}{l}\mathrm{ZrO} \\
(44 \mathrm{~nm})\end{array}$ & $\begin{array}{l}\mathrm{ZrO}_{2} \\
(105 \mathrm{~nm})\end{array}$ \\
\hline$\varphi$ & $\eta_{r}$ & $\eta_{r}$ & $\varphi$ & $\eta_{r}$ & $\eta_{r}$ \\
\hline 0.01 & 1.059 & 1.090 & 0.02 & 1.170 & 1.135 \\
\hline 0.02 & 1.134 & 1.180 & 0.04 & 1.321 & 1.237 \\
\hline 0.04 & 1.357 & 1.570 & 0.06 & 1.589 & 1.519 \\
\hline 0.06 & 1.600 & 1.910 & 0.08 & 2.185 & 2.109 \\
\hline
\end{tabular}

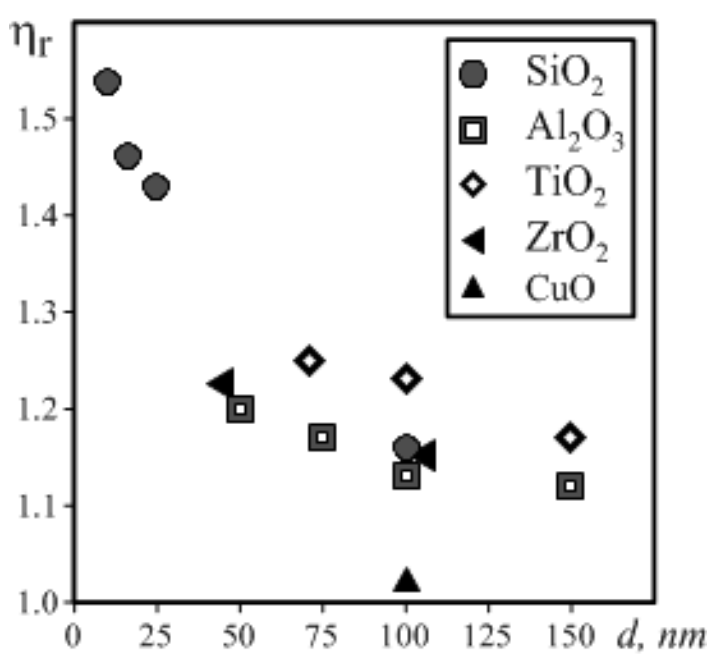

Fig. 3. Relative viscosity coefficient of water based nanofluids versus nanoparticle diameter.

that the viscosity of nanofluids decreases with increasing nanoparticle size.

As already noted above, the viscosity of nanofluids may depend not only on the size but also on the material of nanoparticles. This dependence is clearly seen in Fig. 3 for nanofluids with $100 \mathrm{~nm}$ diameter particles. Previously, this dependence has been detected first using the molecular dynamics method [26] and then experimentally [27]. These differences increase with increasing particle concentration (see Fig. 4).

The temperature dependence of the viscosity of a nanofluid is its important thermophysical characteristic. In liquids, in contrast to gases, the viscosity decreases with increasing temperature. In almost all studies where this dependence has been studied, the viscosity of nanofluids indeed decreases with increasing temperature. The bibliography of papers dealing with the temperature dependence of nanofluid viscosity contains about one hundred titles, some of which can be found in reviews [11, 13]. The temperature dependences of viscosity obtained in all studies are fairly typical. As an example, Fig. 5 shows temperature dependences of viscosity for ethylene glycol based nanofluid [28] with silicon dioxide nanoparticles. At all nanoparticle concentrations, the nanofluid viscosity decreases with increasing temperature. Many different correlations have been proposed to describe the temperature dependence of nanofluid viscosity, but they are not universal and vary substantially as a function of nanoparticle 


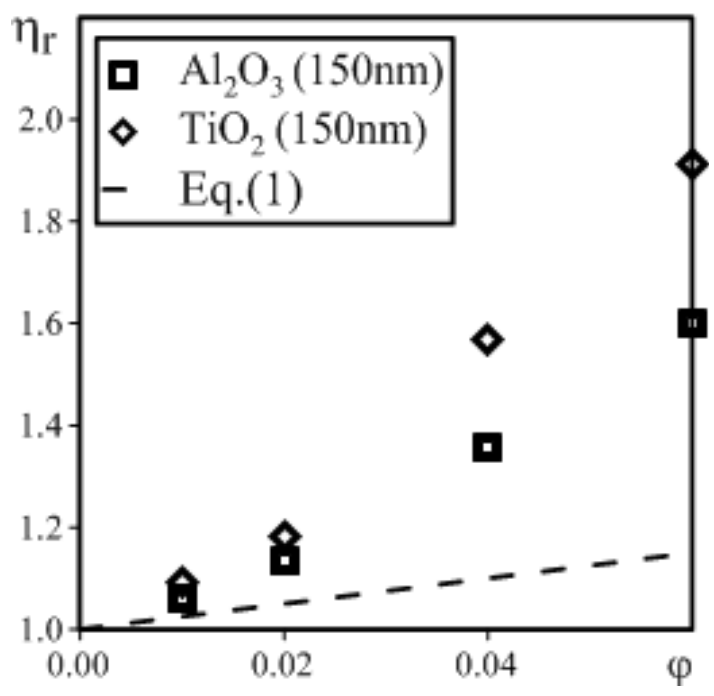

Fig. 4. Relative viscosity coefficiemt of water based nanofluids versus the volume particle concentration.

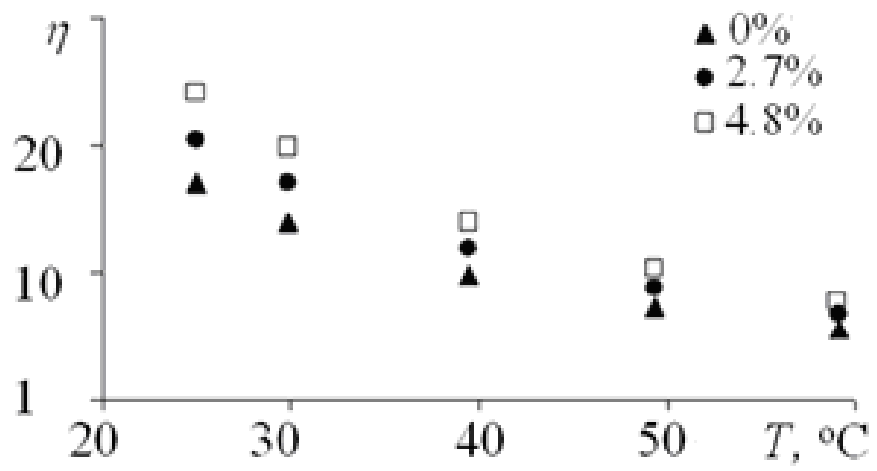

Fig. 5. Temperature dependence of the viscosity coefficient of a nanofluid with $28.3 \mathrm{~nm}$ diameter particles of $\mathrm{SiO}_{2}$ for various volume concentrations.

material, size, and concentration and base fluid viscosity. However the relative viscosity coefficient is practically constant in all known measurements if the particle concentrations are not too large $[14,16,23,28]$.

Thus, the known experimental data and the results of molecular dynamic simulation indicate that the viscosity of nanofluids is significantly higher than the viscosity of ordinary coarse dispersed fluids. At low and moderate concentrations of nanoparticles, it can be described by relation (3). However, the coefficients included in this formula are also a function of nanoparticle material and size. What is the reason for this? There are three main reasons. The first is that in fluids with short-range order and quasi-bound molecules, one of the main mechanisms of momentum transfer involves the destruction of the short-range order. How does the presence of nanoparticles influence the short-range order in fluids? Molecular dynamics simulations [29] have shown that nanofluids are more ordered than the base fluid. The degree of order of the fluid increases with increasing particle concentration and decreasing particles size. An increase in the degree of order of the fluid leads to an increase in its effective viscosity.

In a dispersed fluid with macroscopic particles at volume concentrations $\varphi \sim 10^{-3}$ or less, the distances between particles are large so that their interaction can be neglected. In this case, the viscosity of the dispersed fluid is described by formula (1). On the other hand, it is easy to show that at such concentrations the average distance between nanoparticles is nearly always of the order of their size, and interparticles interaction should be taken into account even at these low concentrations. In formula for ordinary dispersed fluids, the coefficient $k$ in Eq. (2) takes into account the mutual effect of nanoparticles on the nanoparticle-induced perturbations of the velocity field of the dispersed fluid. Therefore, for nanofluids with low nanoparticle concentrations (in the linear approximation for concentration!), the viscosity should be expressed as $\eta=\eta_{0}[1+(2.5+k) \varphi]$. It is this increase in the viscosity of nanofluids with low volume concentrations of nanoparticles that is observed in experiments.

Finally, molecular dynamics simulations have shown [30] that the increased viscosity of nanofluids is due mainly to nanoparticle-molecule interactions and the correlation between molecule-molecule interactions and molecule-nanoparticle interactions. Qualitatively, the dependence of these contributions on the particles size is easy to estimate. Let there be particles with radii $R_{1}$ and $R_{2}$ (for definiteness $R_{2}>R_{1}$ ). For a given volume concentration, the number of these and other particles per unit volume is inversely proportional to the cubes of their radii: $N_{2} / N_{1}=R_{1}^{3} / R_{2}^{3}$. On the other hand, the total scattering cross sections of the base fluid molecules with each other and with nanoparticles are, respectively $S_{1}=N_{1} \pi R_{1}^{2}, S_{2}=N_{2} \pi R_{2}^{2}$. Therefore, the ratio of these cross sections, which determine the magnitude of the potential contributions under discussion, is inversely proportional to their radii: $S_{2} / S_{1}=R_{1} / R_{2}$. These simple estimates show that with increasing particle sizes, the relative magnitude of these contributions will decrease.

\section{Thermal conductivity of nanofluids}

Most expectations have long been associated with various thermal applications of nanofluids. Already the first experimental measurements of the thermal conductivity of nanofluids showed excellent results: the addition of even small (about one percent or less) concentrations of metal nanoparticles increased the thermal conductivity of the base fluid by a few percent or even by a few tens of percent. This has stimulated a number of thermal conductivity measurements in nanofluids; however, the results proved surprisingly controversial. It has been found that the thermal conductivity of nanofluids, just as their viscosity, is not described by classical theories (see the reviews $[31-33])$. In particular, it has been found that the thermal conductivity of nanofluids depends not only on the concentration but also on the size of the particles. However, the nature of this dependence and the magnitude of the thermal conductivity enhancement of nanofluids are 


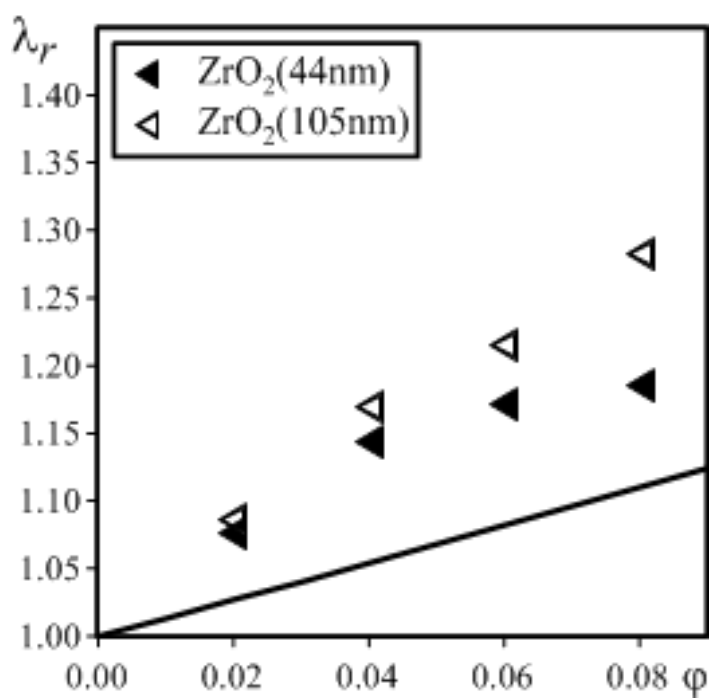

Fig. 6. Dependence of the relative thermal conductivity coefficient of water based nanofluids on $\mathrm{ZrO}_{2}$ particles concentration.

still disputable. Indeed, in [34-36] it was argued that the thermal conductivity coefficient enhances with decreasing particle diameter. It is even asserted [37] that the thermal conductivity increases linearly with decreasing nanoparticle size. On the other hand, the opposite point of view is put forward in [38-40].

Available data on the degree of thermal conductivity enhancement in nanofluids are quite controversial too. Along with the above studies showing a much greater enhancement of the thermal conductivity than predicted by classical theory, there are also assertions that these measurements can be adequately described by Maxwell's theory [41] (see reviews [31, 33, 42]). These conflicting data do not allow formulating neither possible mechanisms nor nanofluid thermal conductivity models, though about a dozen of such models are available in literature (see [43] and the literature quoted there).

Even this brief review shows that systematic measurements of nanofluid thermal conductivity and the determination of influencing parameters are of paramount importance. This section presents the data on the thermal conductivity of nanofluids obtained in our experiments and simulations. The thermal conductivity of nanofluids with various oxide particles was measured using the nonstationary hot-wire method [44]. The error of the measurement of the fluid thermal conductivity coefficient did not exceed $3 \%$. On the other hand, the thermal conductivity of nanofluids with small particles have been studied by the molecular dynamics method.

Firstly, it has been found that as the concentration of nanoparticles increases, the rate of increase in thermal conductivity slows down. This is clearly seen for a water base nanofluid with zirconium dioxide particles in Fig. 6 . The figure shows the dependence of the relative thermal conductivity of nanofluid $\lambda_{r}=\lambda / \lambda_{0}$ ( $\lambda$ and $\lambda_{0}$ are the thermal conductivity coefficients of the nanofluid and based fluid respectively) on the particles concentration.

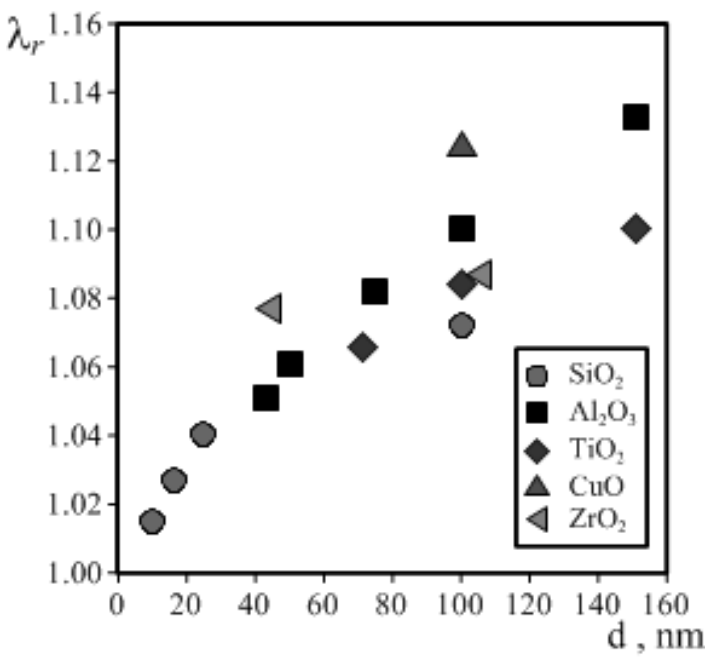

Fig. 7. Relative thermal conductivity coefficient of nanofluids versus particle diameter. The volume concentration of $2 \%$.

The solid line in Fig. 6 corresponds to the Maxwell's formula [41]

$$
\lambda=\lambda_{0}\left[1+\frac{3(1-\varpi) \varphi}{1+2 \varpi-\varphi(1-\varpi)}\right]
$$

where $\varpi=\lambda_{0} / \lambda_{p}, \lambda_{p}$ is the thermal conductivity of the particle material.

Similar behavior of the thermal conductivity of nanofluids has been observed in previous experiments $[45,46]$ and molecular dynamics simulations [47]. At moderate particle concentrations, this dependence can be approximated by the formula

$$
\lambda_{r}=1+b_{1} \varphi-b_{2} \varphi^{2} .
$$

However, the coefficients in this formula are not universal. For a nanofluid containing $150 \mathrm{~nm}$ diameter particles of $\mathrm{Al}_{2} \mathrm{O}_{3}, \lambda_{r}=1+6.42 \varphi-39.5 \varphi^{2}$, for $150 \mathrm{~nm}$ diameter particles of $\mathrm{TiO}_{2}, \lambda_{r}=1+4.82 \varphi-23.1 \varphi^{2}$, for $44 \mathrm{~nm}$ diameter particles of $\mathrm{ZrO}_{2}, \lambda_{r}=1+4.68 \varphi-29.6 \varphi^{2}$, and for $105 \mathrm{~nm}$ diameter particles of $\mathrm{ZrO}_{2}, \lambda_{r}=1+4.55 \varphi-$ $12.7 \varphi^{2}[48]$.

The above-mentioned nonuniversality is primarily due to the fact that the thermal conductivity of nanofluids depends on the particle size $D$. Typical dependences of the relative thermal conductivity of several water-based nanofluids on size of nanoparticles are presented in Fig. 7 (see also the Table 2). It is clearly seen that the larger the particles, the higher the thermal conductivity. How can the dependence of the thermal conductivity on the particle size be described? An analysis [49] of numerous experimental data has been shown that the relative thermal conductivity coefficient of a water-based nanofluid with $\mathrm{Al}_{2} \mathrm{O}_{3}$ particles can be satisfactorily approximated by the formula: $\lambda_{r}=1+A \sqrt{\varphi \tilde{D}}$, where $\tilde{D}=D / d$ ( $d$ is the effective size of the carrier fluid molecule) and constant $A$ should be depended on the nanoparticles material. 
Table 2. Relative thermal conductivity coefficient of water based nanofluids depending on the particle concentration

\begin{tabular}{|l|l|l|l|l|l|}
\hline & $\begin{array}{l}\mathrm{Al}_{2} \mathrm{O}_{3} \\
(150 \mathrm{~nm})\end{array}$ & $\begin{array}{l}\mathrm{TiO}_{2} \\
(150 \mathrm{~nm})\end{array}$ & $\begin{array}{l}\mathrm{ZrO}_{2} \\
(44 \mathrm{~nm})\end{array}$ & $\begin{array}{l}\mathrm{ZrO}_{2} \\
(105 \mathrm{~nm})\end{array}$ \\
\hline$\varphi$ & $\lambda_{r}$ & $\lambda_{r}$ & $\varphi$ & $\lambda_{r}$ & $\lambda_{r}$ \\
\hline 0.01 & 1.059 & 1.048 & 0.02 & 1.077 & 1.087 \\
\hline 0.02 & 1.131 & 1.100 & 0.04 & 1.143 & 1.169 \\
\hline 0.04 & 1.178 & 1.146 & 0.06 & 1.172 & 1.216 \\
\hline 0.06 & 1.240 & 1.206 & 0.08 & 1.185 & 1.283 \\
\hline
\end{tabular}

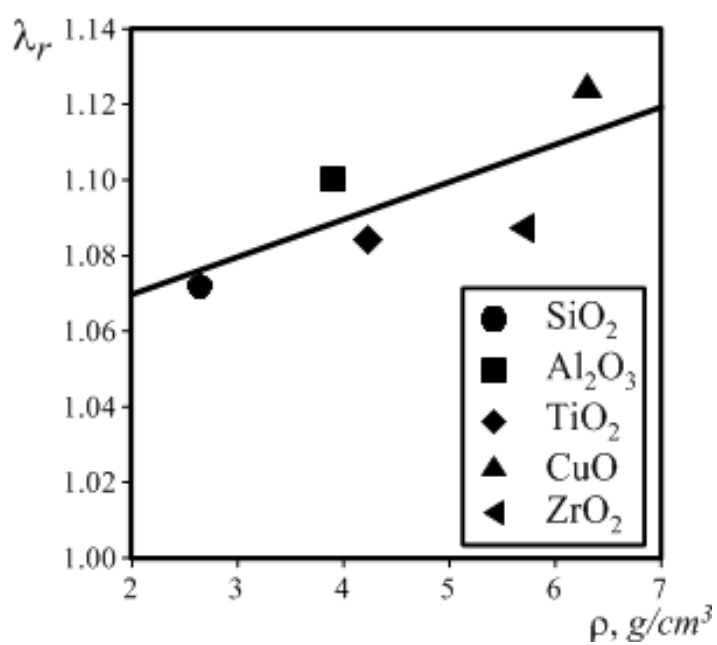

Fig. 8. Relative thermal conductivity coefficient of water based nanofluids depending on density of particle material for volume concentration of $2 \%$ and particle size of $100 \mathrm{~nm}$.

The dependence of the thermal conductivity on material of dispersed particles is important question, of course. Formula (4) gives the dependence of the thermal conductivity on the thermal conductivity of the particle material. However, systematic measurements [48] have shown that there is no correlation between the thermal conductivity of nanofluids and particle material. Nor does the nanofluid thermal conductivity correlate with the thermal diffusivity and specific heat of the particle material.

On the other hand, molecular dynamic simulations [47, $50,51]$ have shown that the higher the nanoparticle density, the higher the thermal conductivity of the nanofluid. Experiments have confirmed this dependence (see Fig. 8), and this dependence is nearly linear.

The constant $A$ in correlation for relative thermal conductivity given above should depend on the density of the particle material. Accordingly, this correlation becomes [48]

$$
\lambda_{r}=1+(0.0193+0.00383 \tilde{\rho}) \sqrt{\varphi \tilde{D}} .
$$

Here $\tilde{\rho}=\rho_{p} / \rho_{f}, \rho_{p}, \rho_{f}$ are the density of the nanoparticle and base fluid materials, respectively. The error of formula (6) is about $3 \%$.

The thermal conductivity of nanofluids decreases with decreasing the particles size. What is the thermal conductivity of nanofluids with the smallest particle? Below answer to this question will obtain by means of the molec- ular dynamics method. The model argon based nanofluids with $\mathrm{Al}$ and $\mathrm{Zn}$ particles were considered. The nanoparticles volume concentration ranged from 1 to $5 \%$. The particle size ranged from 1 to $4 \mathrm{~nm}$.

The method of modeling was described in detailed in paper [26]. Interaction of the carried fluid molecules is described by the Lennard-Jones potential

$$
\Phi_{L J}(r)=4 \varepsilon\left[(\sigma / r)^{12}-(\sigma / r)^{6}\right],
$$

where $r=\left|r_{i}-r_{j}\right|$ is the distance between atoms $i$ and $j$.

Interaction of the carried fluid molecule with nanoparticle is determined by the RK-potential [2]

$$
\begin{gathered}
\Psi(r)=\Psi_{9}(r)-\Psi_{3}(r), \\
\Psi_{i}=C_{i}\left\{\left[\frac{1}{(r-R)^{i}}-\frac{1}{(r+R)^{i}}\right]\right. \\
\left.-\frac{a_{i}}{r}\left[\frac{1}{(r-R)^{i-1}}-\frac{1}{(r+R)^{i-1}}\right]\right\},
\end{gathered}
$$

where $i=9,3, a_{9}=9 / 8, a_{3}=3 / 2, C_{9}=\left(4 \pi \varepsilon_{12} \sigma_{12}^{12}\right) / 45 V_{p}$, $C_{3}=\left(2 \pi \varepsilon_{12} \sigma_{12}^{6}\right) / 3 V_{p}, V_{p}^{-1}=\rho_{p} / m_{p}$. Here $\rho_{p}$ is the density of the nanoparticle material, $m_{p}$ is the mass of the atom of the nanoparticle, $R$ is the radius of the nanoparticle. $\sigma_{i j}, \varepsilon_{i j}$ are the parameters of the interaction potential (7) between a carrier-fluid molecule and an atom of the nanoparticle.

As the nanoparticles interaction potential we use a specially constructed potential [3], which for monodisperse nanoparticles has the form

$$
U(r, R)=U_{7}(r, R)-U_{1}(r, R),
$$

where

$$
\begin{gathered}
U_{7}=\frac{\pi^{2}}{315} \frac{\tilde{\varepsilon} \tilde{\sigma}^{12}}{V_{p}^{2}}\left\{\frac{R^{2}}{r}\left[\frac{1}{(r-2 R)^{7}}+\frac{2}{r^{7}}+\frac{1}{(r+2 R)^{7}}\right]-\right. \\
-\frac{R}{3 r}\left[\frac{1}{(r-2 R)^{6}}-\frac{1}{(r+2 R)^{6}}\right] \\
\left.-\frac{1}{30 r}\left[\frac{1}{(r-2 R)^{5}}-\frac{2}{r^{5}}+\frac{1}{(r+2 R)^{5}}\right]\right\} \\
U_{1}=\frac{2 \pi^{2}}{3} \frac{\tilde{\varepsilon} \tilde{\sigma}^{6}}{V_{p}^{2}}\left[\ln \left(\frac{r^{2}-4 R^{2}}{r^{2}}\right)+2 R^{2}\left(\frac{1}{r^{2}-4 R^{2}}+\frac{1}{r^{2}}\right)\right] .
\end{gathered}
$$

Here $\tilde{\varepsilon}$ and $\tilde{\sigma}$ are the parameters of the Lennard-Jones interaction potential (7) of the nanoparticle atoms (molecules). The parameters of the interaction potential of argon atom were as follows: $\sigma=3.405 \AA$ and $\varepsilon / k_{B}=119.8 \mathrm{~K}$ [20]. The parameters of potentials (8) and (9) were calculated using the following parameters of potential (7): for aluminum, $\sigma=2.551 \AA$ and $\varepsilon / k_{B}=857.6 \mathrm{~K}$, and for zinc, $\sigma=2.46 \AA, \varepsilon / k_{B}=1040 \mathrm{~K}$. The parameters $\sigma_{12}$ and $\varepsilon_{12}$ 
were determined using the simple combinational relations: $\sigma_{12}=\sqrt{\sigma \tilde{\sigma}}$ and $\varepsilon_{12}=\sqrt{\varepsilon \tilde{\varepsilon}}$.

To calculate the thermal conductivity coefficient of nanofluid it is necessary to exclude the heat diffusive flux. Thus the thermal conductivity coefficient is determined as $[53]$

$$
\lambda=\lambda_{t}-\lambda_{d} \equiv \frac{L_{t}}{T^{2}}-\frac{L_{2}^{2}}{L_{22} T^{2}}
$$

Here

$$
\begin{gathered}
L_{t}=\frac{V}{3} \int_{0}^{\tau}\left\langle\mathbf{j}_{Q}(0) \cdot \mathbf{j}_{Q}(t)\right\rangle \mathrm{d} t \\
L_{22}=\frac{V}{3} \int_{0}^{\tau}\left\langle\mathbf{j}_{d 2}(0) \cdot \mathbf{j}_{d 2}(t)\right\rangle \mathrm{d} t \\
L_{2}=\frac{V}{6} \int_{0}^{\tau}\left\langle\mathbf{j}_{Q}(0) \cdot \mathbf{j}_{d 2}(t)\right\rangle \mathrm{d} t+\frac{V}{6} \int_{0}^{\tau}\left\langle\mathbf{j}_{d 2}(0) \cdot \mathbf{j}_{Q}(t)\right\rangle \mathrm{d} t .
\end{gathered}
$$

In these formulas the angular brackets mean averaging over the ensemble, and $\tau$ is the plateau value of the simulation time of integrals [54]. The diffusion flux of nanoparticles (the second component), $\mathbf{j}_{d 2}$ and the heat flux, $\mathbf{j}_{Q}$ are determined by the following relations:

$$
\begin{gathered}
\mathbf{j}_{d 2}(t)=\frac{1}{V} \sum_{\alpha=2, i=1}^{N_{2}} \mathbf{v}_{2, i}(t), \\
\mathbf{j}_{Q}(t)=\mathbf{j}_{Q}^{K}(t)+\mathbf{j}_{Q}^{P}(t)+\mathbf{j}_{Q}^{C}(t) .
\end{gathered}
$$

Here

$$
\begin{gathered}
\mathbf{j}_{Q}^{K}(t)=\frac{1}{V}\left[\frac{m_{1}}{2} \sum_{\alpha=1, i=1}^{N_{1}} \mathbf{v}_{1, i}(t) \mathbf{v}_{1, i}^{2}(t)+\right. \\
\left.+\frac{m_{2}}{2} \sum_{\alpha=2, i=1}^{N_{2}} \mathbf{v}_{2, i}(t) \mathbf{v}_{2, i}^{2}(t)\right] \\
\mathbf{j}_{Q}^{P}(t)=\frac{1}{V}\left[\frac{1}{2} \sum_{\alpha=1, i=1}^{N_{1}} \sum_{\alpha=1, j \neq i} \mathbf{v}_{1, i}(t) \Phi_{11, i j}\left(r_{i j}(t)\right)\right. \\
+\frac{1}{2} \sum_{\alpha=1, i=1}^{N_{1}} \sum_{\alpha=2, j=1}^{N_{2}} \mathbf{v}_{1, i}(t) \Phi_{12, i j}\left(r_{i j}(t)\right) \\
+\frac{1}{2} \sum_{\alpha=2, i=1}^{N_{2}} \sum_{\alpha=1, j=1}^{N_{1}} \mathbf{v}_{2, i}(t) \Phi_{12, i j}\left(r_{i j}(t)\right) \\
\left.+\frac{1}{2} \sum_{\alpha=2, i=1}^{N_{2}} \sum_{\alpha=2 j \neq i} \mathbf{v}_{2, i}(t) \Phi_{22, i j}\left(r_{i j}(t)\right)\right]
\end{gathered}
$$

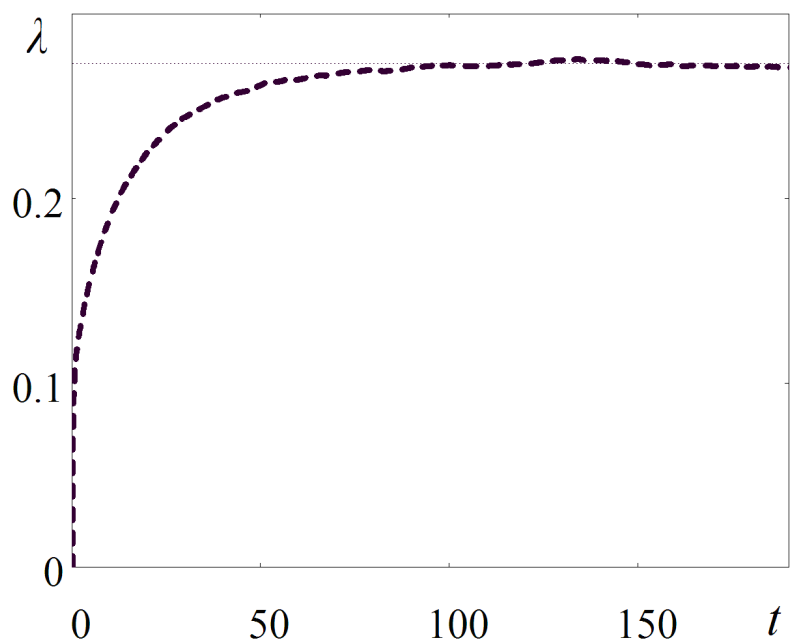

Fig. 9. Evolution of the thermal conductivity coefficient of nanofluid $\lambda(t), \mathrm{W} /(\mathrm{mK}$.

$$
\begin{aligned}
\mathbf{j}_{Q}^{C}(t) & =\frac{1}{V}\left[\frac{1}{2} \sum_{\alpha=1, i=1}^{N_{1}} \sum_{\alpha=1 j \neq i} \mathbf{r}_{i j}(t)\left(\mathbf{F}_{11, i j}(t) \cdot \mathbf{v}_{1, j}(t)\right)\right. \\
& +\frac{1}{2} \sum_{\alpha=1, i=1}^{N_{1}} \sum_{\alpha=2, j=1}^{N_{2}} \mathbf{r}_{i j}(t)\left(\mathbf{F}_{12, i j}(t) \cdot \mathbf{v}_{2, j}(t)\right) \\
& +\frac{1}{2} \sum_{\alpha=2, i=1}^{N_{2}} \sum_{\alpha=1, j=1}^{N_{1}} \mathbf{r}_{i j}(t)\left(\mathbf{F}_{12, i j}(t) \cdot \mathbf{v}_{1, j}(t)\right) \\
& \left.+\frac{1}{2} \sum_{\alpha=2, i=1}^{N_{2}} \sum_{\alpha=2 j \neq i} \mathbf{r}_{i j}(t)\left(\mathbf{F}_{22, i j}(t) \cdot \mathbf{v}_{2, j}(t)\right)\right]
\end{aligned}
$$

Here the index $\alpha=1$ refers to the molecules of argon, 2 - to nanoparticles, $m_{i}$ is the mass of the particle (molecule or nanoparticle), $V$ is the volume of the system, $T$ is temperature of the fluid, $\Phi_{11, i j}$ is the interaction potential of the based fluid molecules, $\Phi_{12, i j}$ is the interaction potential between base fluid molecules and nanoparticles, $\Phi_{22, i j}$ is the interaction potential of the particles, and $\mathbf{F}_{\alpha \beta, i j}$ are the appropriate forces. $N_{1}, N_{2}$ are the number of argon molecule and nanoparticles respectively.

The simulated thermal conductivity coefficient is a function of time. The actual value of this coefficient is obtained when the integration time reaches the plateau value of $\tau$ after which it ceases to change. The evolution of the function (10) for a nanofluid with Zn particles of size of $2 \mathrm{~nm}$ is presented in Fig. 9. Here the volume concentration is equal to $4.2 \%$. The time is measured in units $\tau=\sigma / c$, where $c$ is the thermal velocity of the carrier fluid molecules.

Typical dependence of the thermal conductivity coefficient of nanofluid considered above on volume concentration of nanoparticles is presented in Fig. 10. Here the triangles correspond to simulated data and line 2 to formula (4). The thermal conductivity of considered nanofluid is 


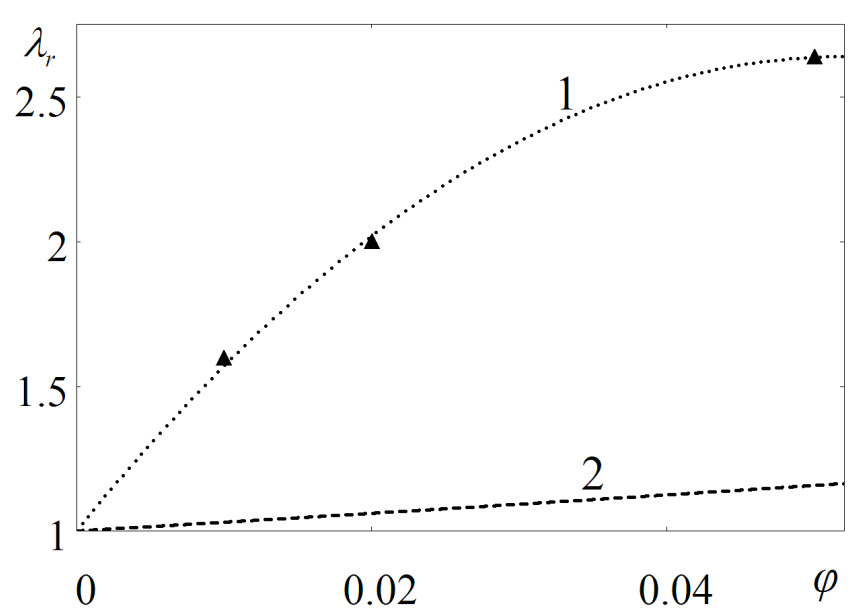

Fig. 10. Relative thermal conductivity coefficient of the Ar-Zn nanofluid versus volume concentration of the nanoparticles $(D$ $=2 \mathrm{~nm})$.

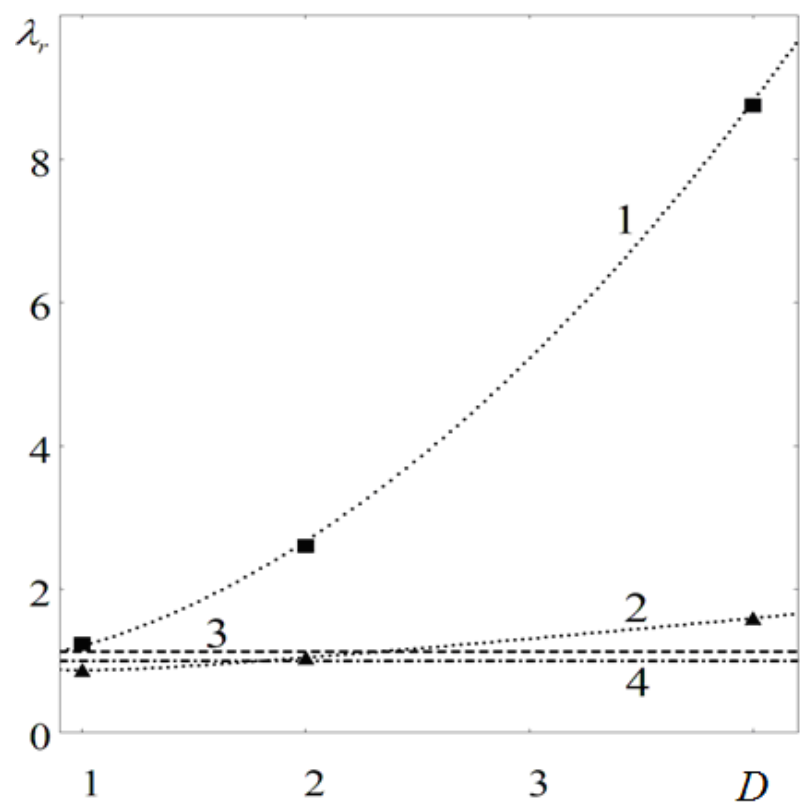

Fig. 11. Relative thermal conductivity coefficient of nanofluids Ar-Zn and Ar-A versus the diameter (nm) of nanoparticles.

much higher than the thermal conductivity of both the base fluid and coarse dispersed fluids. The line 2 is described by the formula (5) with the following constants: $b_{1}=63.1, b_{2}=607.9$.

As follows from (10), the thermal onductivity coefficient of a nanofluid is a superposition of two terms. The second term occurs only in a nanofluids. However its value is not large (maximum magnitude is not exceed the five percents) and this contribution decreases with increasing the concentration of the particles.

The dependence of the thermal conductivity on size of nanoparticles depends significantly on their material. Figure 11 shows curves of the thermal conductivity of nanofluids versus nanoparticle diameter for two nanofluids with
Zn (squares) and Al (triangles) particles. The nanoparticle volume concentration is the same in both cases (4.2\%). Lines 3 and 4 correspond to the thermal conductivity of coarse dispersed fluid (formula (4)) and pure argon, respectively. The data shown in the figure differ greatly from each other. The thermal conductivity of the nanofluid Ar$\mathrm{Zn}$ is much higher than the Maxwell's value. In contrast, the thermal conductivity of the nanofluid with $1 \mathrm{~nm}$ aluminum particles is even lower than the thermal conductivity of the base fluid. However, the thermal conductivity of the nanofluid with $4 \mathrm{~nm}$ particles already exceeds the value given by formula (4). The low thermal conductivity of nanofluids with small particles has previously been experimentally demonstrated (see [48]). However in all cases the thermal conductivity of nanofluid becomes higher than the thermal conductivity of coarse dispersed fluid when the particle size grows.

It is very interesting to analyze the contribution of each term in (10). Such detailed analyze can be found in paper [55]. Here we will make only two remarks. The thermal conductivity of pure fluids is mainly determined by the energy transfer due to molecular collisions. In nanofluids, this energy transfer channel also takes place, but there is a new, more important channel due to mutual motion of molecules and nanoparticles. Diffusion fluxes of molecules and nanoparticles are the most important factor in the increase of the thermal conductivity of nanofluids.

There is no generally accepted model for the thermal conductivity of nanofluids, although many attempts have been made to develop it (see review [42] and references therein). Apparently, this has been done most systematically by Keblinski et al. [46, 58] and Kleinstreuer and Feng [59], who have analyzed the contributions of four possible mechanisms: (i) ballistic phonon heat transport, (ii) the formation of a fluid layer of increased thermal conductivity around nanoparticles, (iii) nanoparticle Brownian motion, and (iv) nanoparticle clustering. The contribution of the ballistic phonon mechanism to the thermal conductivity enhancement has been correctly rejected by almost all authors.

As regards nanoparticle clustering, this effect, in principle, could lead to an increase in thermal conductivity. However, clustering would result in the formation of particles with macroscopic sizes in the fluid. This, on the one hand, implies that the thermal conductivity must cease to depend on the nanoparticle size (as in classical theories), and, on the other hand, such particles should be rapidly sedimented. Neither of these has been observed in well-designed experiments. This is also supported by molecular dynamic calculations for hard spheres [50, 51], in which clustering is not possible in principle, but which, nevertheless, show a marked increase in thermal conductivity compared to the Maxwell's theory.

The Brownian motion of nanoparticles has been repeatedly discussed as a mechanism for increasing the thermal conductivity of nanofluids compared to pure fluids. The direct influence of the Brownian motion of nanoparticles on the thermal conductivity of nanofluid is not great, as it is correctly noted in $[46,58]$. However, a nanofluid is 
a binary system of particles, in which there is mutual diffusion of the active components. The resulting fluxes are apparently the main mechanism that governs the thermal conductivity of nanofluids. Due to the diffusive motion of molecules and nanoparticles, the contribution of the transferred kinetic energy fluxes to the thermal conductivity can increase essentially.

\section{Heat transfer coefficient}

This section deals with experimental study of the convective heat transfer of nanofluids in laminar and turbulent regimes of flow. The experimental setup was described in detail in $[56,57]$. It was a closed loop with a circulating coolant. The working fluid was circulated by a pump through the heated measuring section. The heated section was a stainless steel tube $6 \mathrm{~mm}$ in diameter and $1 \mathrm{~m}$ long. The tube was heated by supplying an electric current directly to its wall. Local temperature of the tube was measured with six copper-constantan thermocouples attached to its walls. In addition, the temperature at the inlet and outlet of the heated section was measured using thermocouples. Pressure gradient was measured with a differential pressure gauge. The measurement error was $0.1 \%$ for the pressure gradient and about $1 \%$ for temperature. Heat transfer was studied for several water-based nanofluids with $\mathrm{CuO}(55 \mathrm{~nm}), \mathrm{TiO}_{2}(100 \mathrm{~nm}), \mathrm{ZrO}_{2}(105$ $\mathrm{nm}), \mathrm{SiO}_{2}(10,16,25,100 \mathrm{~nm})$, and $\mathrm{Al}_{2} \mathrm{O}_{3}(10,25,100$ $\mathrm{nm})$ particles. The particle concentration was varied from 0 to $2 \%$.

The measurements showed that the addition of nanoparticles always significantly increased the local and the average heat transfer coefficient of the fluids in laminar flow. Typical dependences of the average heat transfer coefficient $\left(\alpha, \mathrm{W} / \mathrm{m}^{2} \mathrm{~K}\right)$ for nanofluids with different particle concentrations on the Reynolds (Re) number are shown in Fig. 12. Here $\alpha=G C_{p}\left(T_{i}-T_{o}\right) S^{-1}\left(\bar{T}_{w}-\bar{T}\right)^{-1}$, where $C_{p}$ is the specific heat of the heat transfer agent, $S$ is the area of the lateral surface of the channel, $T_{o}$ and $T_{i}$ are the temperatures of the fluid at the outlet and inlet of the channel, $\bar{T}=\left(T_{i}+T_{o}\right) / 2, T_{w}$ is the temperature of the channel wall. The temperature $\bar{T}_{w}$ is the arithmetic average temperature of the channel wall, obtained by averaging the data for the six thermocouples data.

The heat transfer coefficient of nanofluids with $\mathrm{CuO}$ nanoparticles increases rapidly with increasing their concentration: for the one-percent nanofluid, it is more than $40 \%$ higher than that for water at practically all Reynolds numbers $(\mathrm{Re})$, and at a particle concentration equal to $2 \%$, it is more than twice that for water. Naturally, with a decrease in the concentration of nanoparticles, this effect decreases monotonically. At low flow rates when the laminar flow certainty occurs in both nanofluids considered and in water, the degree of this increase grows almost proportionally to the volume concentration of nanoparticles, that is, $\Delta \alpha=\left(\alpha-\alpha_{0}\right) / \alpha_{0} \sim \varphi$, where $\alpha_{0}$ is the heat transfer coefficient of water.

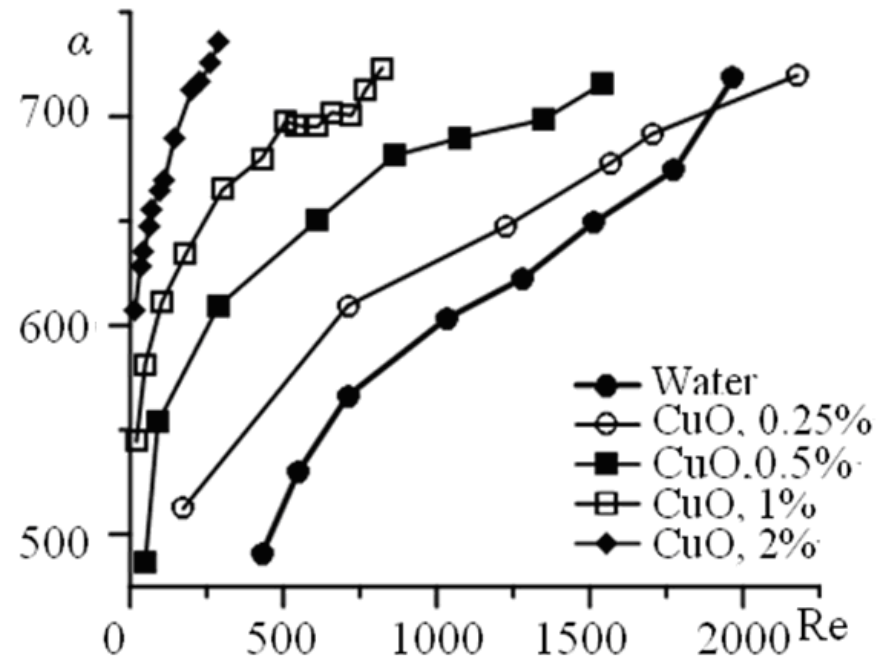

Fig. 12. Average heat transfer coefficient versus Reynolds number.

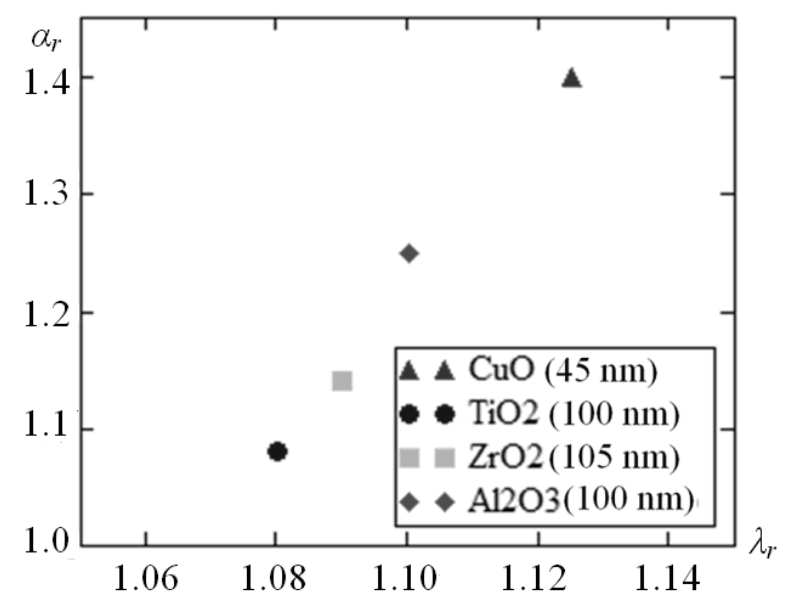

Fig. 13. Relative heat transfer coefficient versus relative thermal conductivity of the water based nanofluids for different nanoparticle materials at constant Reynolds number.

If the value of the Reynolds number is above 2000, the laminar-turbulent transition takes place in water, which intensifies its heat transfer.

In laminar flow, the heat transfer coefficient increases almost linearly with increasing thermal conductivity of nanofluid. This is illustrated in Fig. 13, which shows the dependence of the relative heat transfer coefficient $\alpha_{r}=$ $\alpha / \alpha_{0}$ on the relative thermal conductivity.

In turbulent flow, the situation is much more complicated. Typical dependences of the average heat transfer coefficient for nanofluids with silicon oxide particles with an average particle size of $25 \mathrm{~nm}$ are presented in Fig. 14. Here the maximum particle concentration was $2 \%$. In all cases, the heat transfer coefficient of the nanofluids is higher than that of water and increases with increasing nanoparticle concentration. The extent of this increase is practically proportional to the volume concentration of nanoparticles, just as in laminar flow. The heat transfer 


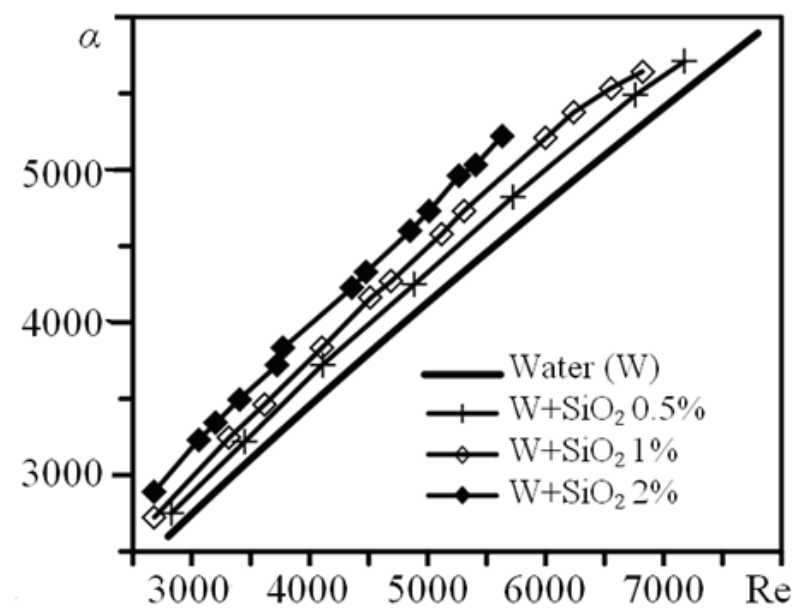

Fig. 14. Heat transfer coefficient of nanofluids versus Reynolds number for different concentrations of $25 \mathrm{~nm}$ particle.

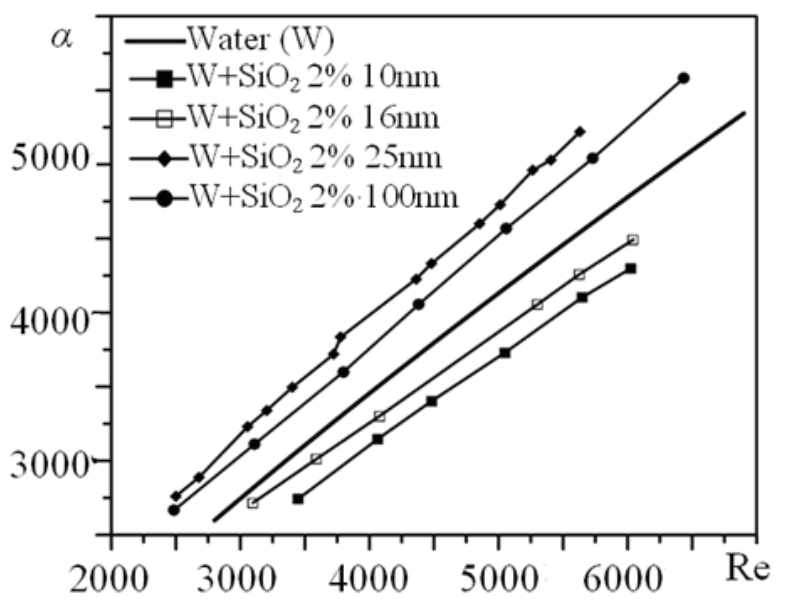

Fig. 15. Heat transfer coefficient of nanofluids versus Reynolds number for different average particle sizes and fixed particle concentrations $(2 \%)$.

coefficient of the two-percent nanofluid is more than $15 \%$ higher than that for water.

Since the nanofluid transport coefficients are a function of particle size, the heat transfer coefficient also depends on the particle size. Figure 15 shows the dependence of the average heat transfer coefficient on the Reynolds number for water-based nanofluids with silicon oxide particles of four sizes: 10, 16, 25, and $100 \mathrm{~nm}$. In all cases, the volume concentration was $2 \%$. The heat transfer coefficient at a fixed Reynolds number is maximal for a nanofluid with $25 \mathrm{~nm}$ diameter particles. Here it is about $15-20 \%$ higher than the corresponding values for water. For the nanofluid with $100 \mathrm{~nm}$ diameter particles, the heat transfer coefficient enhancement is approximately $10 \%$. On the other hand, the heat transfer coefficient of nanofluids with 10 and $16 \mathrm{~nm}$ diameter particles is lower than that of water. This is due to the fact that the heat transfer of a nanofluid in turbulent flow depends not only on its thermal conductivity, but also on viscosity. Therefore, if the increase in the thermal conductivity of the heat transfer fluid due to

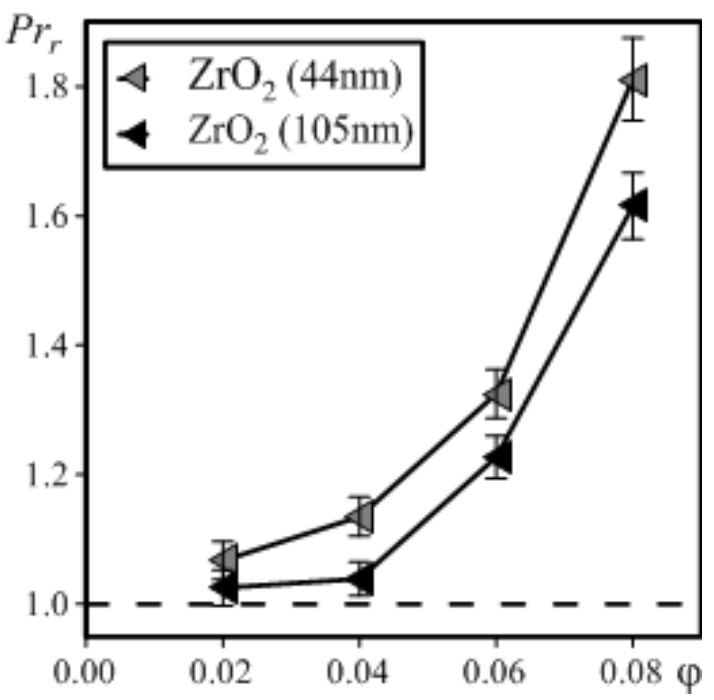

Fig. 16. Relative Prandtl number of a water based nanofluid versus the concentration of $\mathrm{ZrO}_{2}$ nanoparticles.

the nanoparticles is significantly lower than the increase in its viscosity, there may be a reduction in the heat transfer coefficient. This is the key difference between turbulent and laminar heat transfer for nanofluids.

In practice, all applications and laboratory investigations of nanofluids deal with their flows. Description of the nanofluid flows, as well as usual fluids, typically employs well-known similarity criteria, such as Reynolds, Prandtl, Nusselt numbers and so on. The unusual thermophysical properties of nanofluids do not always permit the use of standard similarity parameters. For example, the Prandtl number $\operatorname{Pr}=\left(C_{p} \mu\right) / \lambda$ is a complex function of particle concentration, size, and material. Simple variation of this parameter in modeling nanofluid flows may be useless because the simulated flow property at each point will just correspond to different nanofluids. This is illustrated in Fig. 16, which shows the dependence of the relative Prandtl number $\operatorname{Pr}_{r}=\operatorname{Pr} / \operatorname{Pr}_{0}\left(\operatorname{Pr}_{0}\right.$ is the Prandtl number of the base fluid) on the nanoparticle concentration for two nanofluids with $\mathrm{ZrO}_{2}$ particles [60].

\section{Conclusion}

Thus, nanofluids are not conventional suspensions. Their thermophysical properties are not described by the classical theories. Today we can say with confidence that the viscosity of nanofluids is significantly higher than the viscosity of conventional coarse dispersed fluids. This conclusion is confirmed by recent experiments and molecular dynamics simulationThis. In contrast to the viscosity of coarse dispersed fluid, the viscosity of nanofluids depend not only on the particle concentration but also on their size and material. It is clear the reasons of such behavior. The main reason for this dependence is that the nanofluid is much more ordered (in the sense of short-range order) than the base fluid. The higher the particle concentration 
and less their size, the more ordered the fluid. The dependence of the nanofluid viscosity on the particle material is a more subtle property. For nanofluids with very small particles, this property is fairly easy to explain. However, in experiments this dependence has also been observed for nanofluid with large particles. Therefore, this problem requires further research. Nevertheless, today one can confidently predict the viscosity of practically any preassigned nanofluid.

The thermal conductivity of nanofluids (similarly to their viscosity) is not described by classical theories, in particular, by the Maxwell's formula (or its generalizations). The thermal conductivity of nanofluid also depend on size and material of nanoparticles. As a rule the thermal conductivity far exceed the value given by the Maxwell's formula. It increases with increasing particle concentration, reaches a certain maximum, and then varies only slightly. In contrast to classical theories, the thermal conductivity of nanofluids depends on the nanoparticle size and increases with its increase. However, there may be situations where the thermal conductivity of nanofluids with a small particles is not higher (or lower) than the values predicted by the Maxwell's theory. We can not predict and explain the reason of this behavior. However, with further increase in nanoparticle size, the thermal conductivity of nanofluids consistently increases and becomes higher than the values predicted by the Maxwell's formula.

The effect of the thermal conductivity enhancement is determined not only by the nanoparticle size, but also by the density of the nanoparticle material. In this regard, we note that the mass concentration of nanoparticles in nanofluids increases dramatically with increasing density of the particle material. Thus, the nanofluid thermal conductivity enhancement over the value for the base fluid is greater, the higher the mass fraction of nanoparticles.

The heat transfer coefficient of nanofluids is determined by the particles concentration. It is shown that the use of nanofluids can lead to a considerable increase in the heat transfer coefficient, as compared with that for the base fluid. However, the effect obtained also depends on the nanoparticle size and material. Because of this, under certain conditions the heat transfer coefficient of a nanofluid can turn out to be lower than that of the base fluid. This is due, in particular, to the opposite influence of the nanoparticle size on the nanofluid viscosity and thermal conductivity: the nanofluid viscosity increases with the nanoparticle size, whereas its thermal conductivity decreases. In addision the enchsansment of the heat transfer coefficient depends on the nanofluid flow mode (laminar or turbulent). This provides the understanding of the rather contradictory experimental data available in the literature.

This work was supported by the Russian Foundation for Basic Research (Grants \#17-01-00040, \#17-58-45032), Government of Krasnoyarsk Territory, Krasnoyarsk Region Science and Technology Support Fund to the research project \#16-48-243061, the Russian Science Foundation (Project \#17-79-20218) and Project of the President of Russian Federation (Project \# RF HS-8788.2016.1).

\section{Authors contributions}

VYaR performed a molecular dynamics simulation of the viscosity and thermal conductivity of nanofluids. Viscosity measurements were performed by both co-authors. While measurements of the thermal conductivity and the heat transfer coefficient are performed by the AVM.

\section{References}

1. D.C.Rapaport, The Art of Molecular Dynamics Simulation (Cambridge University Press, Cambridge, 1995) 549

2. V.Ya. Rudyak, S.L. Krasnolutskii, Doklady Physics 46, (2011) 897.

3. V.Ya. Rudyak, S.L. Krasnolutskii, D.A. Ivanov, Doklady Physics 57, (2012) 33.

4. A. Einstein, Annalen der Physik 19, (1906) 289.

5. M. Mooney, J. Colloid Sci 6, (1951) 162.

6. I.M. Krieger, T.J. Dougherty, J. Rheology 3, (1959) 137.

7. N.A. Frankel, A. Acrivos, Chem. Eng. Sci 22, (1967) 847.

8. I.M. Krieger, Adv. Colloid Interface Sci 3, (1972) 111.

9. G.K. Batchelor, J. Fluid Mech 83, (1977) 97.

10. A. Acrivos, E.Y. Chang, Phys. Fluids 29, (1986) 459.

11. S.Sh. Hosseini, A. Shahrjerdi, Y. Vazifeshenas, Australian J. Basic Appl. Sci 5, (2011) 417.

12. D. C. Venerus, et al, Applied Rheology 20, (2010) 44582.

13. M. Mahbubul, R. Saidur, M.A. Amalina, Int. J. Heat Mass Transfer 55, (2012) 874.

14. H. Chen, et al., Chemical Physics Letters 444, (2007) 333.

15. P. K. Namburu, et al., Micro Nano Lett. 2, (2007) 67.

16. C.T. Nguyen et al., Int. J. Therm. Sci. 47, (2008) 103.

17. F.S. Oueslati, R. Bennace, Nanoscale Research Lett 6, (2011) 222.

18. B.C. Pak, Y. I. Cho, Experimental Heat Transfer 11, (1998) 151.

19. X. Wang, X. Xu, S. U. S. Choi, Thermophysics and Heat Transfer 13, (1999) 474.

20. V.Ya. Rudyak, et al. Preprint (NSUACE, Novosibirsk, 2006)

21. V.Ya. Rudyak, A.A. Belkin, V.V. Egorov, Technical Physics 54, (2009) 1102.

22. P. Prasher, D. Song, Wang. Appl. Phys. Lett 89, (2006) 133108.

23. Y. He, et al., Int. J. Heat Mass Transfer 50, (2007) 2272.

24. E.V. Timofeeva, et al., Nanotechnology 21, (2010) 215703.

25. V.Ya. Rudyak, et al., Doklady Physics 58, (2013) 173.

26. V.Ya. Rudyak, S.L. Krasnolutskii, Phys. Letters A 378, (2014) 1845.

27. V.Ya. Rudyak, et al., Doklady Physics 61, (2016) 152.

28. V.Ya. Rudyak, S.V. Dimov, V.V. Kuznetsov, Technical Physics Letters 39, (2013) 779.

29. V.Ya. Rudyak, Advances in Nanoparticles 2, (2013) 266.

30. V.Ya. Rudyak, S.L Krasnolutskii, Technical Physics 60, (2015) 798.

31. X-Q.Wang, A.S. Mujumdar, Int. J. Thermal Sciences 46, (2007) 1.

32. W. Yu, et al., Heat Transfer Eng 5, (2008) 432.

33. K. Kleinstreuer, F. Yu, Nanoscale Research Letters 6, (2011) 1.

34. C.H. Chon, Appl. Phys. Lett 87, (2005) 153107.

35. C.H. Li, G.P. Peterson, Appl. Phys. 101, (2007) 044312. 
36. H.A. Mintsa, et al., Int. J. Thermal Sci 48, (2009) 363.

37. S.H. Kim, S.R. Choi, D. Kim, ASME J. Heat Transfer 129, (2007) 298.

38. G. Chen, et al., Nanopart. Res. 10, (2008) 1109.

39. M.P. Beck, et al., Nanopart. Res 11, (2009) 1129.

40. E.V.Timofeeva, et al., Nanotechnology 21, (2010) 215703.

41. J.C. Maxwell, A treatise on electricity and magnetism (Clarendon Press, Oxford, 1881)

42. P.M. Kumar, et al., Eng. J 19, (2015) 67.

43. W. Rashmi, et al., Materials Research Express 1, (2014) 032001.

44. A.V. Minakov, et al., Eng. Phys. Thermophysics 88, (2015) 149.

45. H.T. Zhu, et al., Phys. Chem. C. 111, (2007) 1646.

46. V.Ya. Rudyak, A.A. Belkin, E.A. Tomilina, Proceedings of the 3d European Conf. Microfluidics. 152, (2012) 8.

47. P. Keblinski, R. Prasher, J. Eapen, Nanopartarticles Res. 10, (2008) 1089.

48. M.I. Pryazhnikov, et al., Int. J. Heat Mass Transfer 104, (2017) 1275.

49. D. ?eotto, V.Ya. Rudyak, Colloid J. 78, (2016) 509.

50. V.Ya. Rudyak, A.A. Belkin, Nanosystems: Phys., Chem., Math 1, (2010) 156.

51. V.Ya. Rudyak, A.A. Belkin, E.A. Tomilina, Technical Phys. Letters 36, (2010) 49.

52. J.O. Hirschfelder, Ch.F. Curtiss, R.B. Bird, Molecular theory of gases and liquids (Wiley, New York, 1954)

53. D. Zubarev, Nonequilibrium statistical thermodynamics (Consultants Bureau, New York, 1974)

54. V.Ya. Rudyak, et al., High Temperature 46, (2008) 30.

55. V.Ya. Rudyak, S.L Krasnolutskii, Technical Phys 62, (2017) 894.

56. A.V. Minakov, et al., High Temperature 53, (2015) 246.

57. A.V. Minakov, et al., Appl. Thermal Engineering 88, (2015) 140.

58. P. Keblinski, et al., Int. J. Heat Mass Transfer 45, (2002) 855.

59. C. Kleinstreuer C., Y. Feng, Nanoscale Research Letters 6, (2011) 229

60. V.Y. Rudyak, A.V. Minakov, M.I. Pryazhnikov, Technical Phys. Letters 43, (2017) 23. 